\title{
Blood pressure reactivity to mental stress is attenuated following resistance exercise in older hypertensive women
}

This article was published in the following Dove Press journal:

Clinical Interventions in Aging

15 May 2017

Number of times this article has been viewed

\author{
Rafael Gauche' \\ Ricardo M Lima ${ }^{1,2}$ \\ Jonathan Myers ${ }^{2}$ \\ André B Gadelha' \\ Silvia GR Neri' \\ Claudia LM Forjaz ${ }^{3}$ \\ Lauro CVianna' \\ 'Faculty of Physical Education, \\ University of Brasília, Brasília, Brazil; \\ ${ }^{2}$ Cardiology Division, Veterans Affairs \\ Palo Alto Health Care System and \\ Stanford University, Palo Alto, CA, \\ USA; ${ }^{3}$ School of Physical Education \\ and Sport, University of São Paulo, \\ São Paulo, Brazil
}

Purpose: This study aimed to investigate the effects of resistance exercise (RE) on autonomic control and blood pressure (BP) reactivity during mental stress (MS) in treated older hypertensive women.

Methods: Ten older hypertensive women (age $=71.1 \pm 5.5$ years; body mass index $=24.2 \pm 3.9$; mean $\mathrm{BP}[\mathrm{MBP}]=85.4 \pm 3.5$ ) underwent a protocol consisting of $\mathrm{BP}$ and heart rate variability (HRV) output assessments at baseline and during MS, and these measurements were taken before and 60 minutes after two bouts of RE (traditional and circuit). MS was induced through a computerized 3-minute Stroop color-word test before and 1 hour after each exercise session; BP was measured every minute during MS, and HRV was monitored as a measure of cardiac autonomic control.

Results: A significant effect of time on systolic BP $(\Delta \mathrm{pre}=17.4 \pm 12.8$ versus $\Delta$ post $=12.5 \pm 9.6$; $P=0.01)$, diastolic BP $(\Delta \mathrm{pre}=13.7 \pm 7.1$ versus $\Delta$ post $=8.8 \pm 4.5 ; P=0.01)$, and MBP $(\Delta \mathrm{pre}=14.0 \pm 7.7$ versus $\Delta$ post $=9.3 \pm 5.4 ; P<0.01)$ after RE was observed, with no differences between the two sessions. In addition, a significant effect of time on log-normalized low-frequency component of HRV ( $\mathrm{ms}^{2} ; 5.3 \pm 0.8$ pre-exercise MS versus $4.8 \pm 1.0$ baseline value; $P=0.023$ ) was also observed, showing a significant change from baseline to MS before RE, but not after RE sessions. These results may be related to a lessened RE-mediated cardiac sympathetic activity during MS.

Conclusion: RE is an effective tool to reduce BP reactivity to MS, which could therefore be associated with an acute reduction in cardiovascular risk. This result presents relevant clinical implications, combining previous evidence that recommends this exercise modality as an important component of an exercise program designed for the older and hypertensive subjects.

Keywords: resistance training, psychological stress, aging, hypertension, autonomic nervous system

\section{Introduction}

Hypertension (HTN) is a growing health problem that plays an important role in the development of cardiovascular disease. ${ }^{1}$ The prevalence of HTN increases with increasing age; currently, more than half of older individuals are affected. ${ }^{2}$ Moreover, although blood pressure (BP) is typically lower in women, postmenopausal women have a higher prevalence of HTN when compared with age-matched men. ${ }^{3}$ The management of HTN in older individuals is clearly relevant given that its prevalence and related complications are expected to increase with aging population, especially in older women. Various lifestyle modifications have been demonstrated to positively influence BP and thus is thought to be the first-line treatment. ${ }^{1}$ In particular, physical exercise is an effective treatment strategy for individuals with HTN. ${ }^{4}$
Correspondence: Ricardo M Lima Universidade de Brasília, Campus Universitário Darcy Ribeiro, Faculdade de Educação Física, Brasília, DF 70910-900, Brazil

Tel +55 6I 31072512

Email ricardomoreno@unb.br 
Hypertensive patients, as well as the general population, are exposed to mental and psychological stress in day-to-day life, imposing an increased load on the cardiovascular system. ${ }^{5}$ Specifically, BP rise to a given stress stimulus in relation to baseline values has been referred to as BP reactivity. An augmented BP response to mental stress (MS) has been reported to be associated with future HTN development ${ }^{6}$ and to predict carotid atherosclerosis in women. ${ }^{7}$ Moreover, during MS, individuals with HTN have higher BP values when compared with their age-matched normotensive counterparts ${ }^{8,9}$ and have impaired endothelial-dependent vasodilation. ${ }^{10}$ These findings indicate that hypertensive subjects are at an increased risk of cardiovascular events and have greater susceptibility to vascular damage when exposed to frequent stressful situations.

Importantly, several reports have shown that the BP response to MS is attenuated after a single session of aerobic exercise $^{11-13}$ (the rhythmical contraction and relaxation of large muscle groups over a prolonged time) in healthy subjects. Furthermore, these findings have been extended to individuals with metabolic syndrome ${ }^{14}$ and to patients with HTN, ${ }^{15,16}$ which has important clinical implications. In addition, it has been demonstrated that exercise promotes a reduction in vascular reactivity among individuals with a family history of HTN ${ }^{17} \mathrm{Few}$ studies have been conducted to elucidate mechanisms associated with the acute reduction in $\mathrm{BP}$ reactivity after exercise. In this regard, Brownley et al ${ }^{12}$ suggested that reduced BP reactivity to MS after an aerobic exercise session is associated with a decrease in sympathetic drive, whereas Ebbesen et $a 1^{18}$ observed the maintenance of catecholamine levels in response to MS. The mechanisms underlying attenuated BP responses to MS following a single bout of exercise require further exploration.

In fact, most physical activity guidelines for hypertensive individuals traditionally recommend aerobic activities such as cycling, walking, and jogging. ${ }^{4}$ However, in addition to the well-documented positive effects of resistance training on muscle mass, strength, and function, more recent studies support many benefits of this training modality on cardiovascular health, such as positive changes in cardiovascular system structure and function and reduction in resting BP. ${ }^{19}$ In addition, a single session of resistance exercise (RE) may be effective in reducing BP levels below resting levels. ${ }^{20}$ Thus, RE has been used as an important component of physical activity programs for BP control. ${ }^{4}$ However, little is known regarding the effect of a single bout of RE on BP reactivity to MS. In studies on RE among hypertensive subjects, exercise sessions are typically implemented using either a traditional (TR) multiple-set or a circuit-based (CI) regimen. ${ }^{21,22}$ Thus, in addition to examining the effects of $\mathrm{RE}$ on BP reactivity to MS, it would be informative to compare both the TR and CI approaches. Therefore, the aim of the present study was to investigate the acute effects of TR and CI RE sessions on $\mathrm{BP}$ reactivity to MS in treated older hypertensive women. Autonomic control was also examined in an effort to elucidate potential mechanisms. Based on the literature, ${ }^{23}$ the hypothesis that both these RE arrangements would acutely attenuate BP reactivity to MS and thus provide additional support for exercise recommendation was raised.

\section{Methods \\ Study design}

In order to examine the acute effects of different RE modes on BP reactivity and autonomic modulation in older hypertensive women, each volunteer underwent six visits to the laboratory. During the first visit, the subjects completed face-to-face questionnaires addressing their medical history and lifestyle habits. Resting BP was measured by using an oscillometric device (Microlife BP 3AC1-1; Microlife USA Inc., Dunedin, FL, USA) at least two times, after 5 minutes of seated rest and with an interval of 5 minutes between readings. If a difference $>5 \mathrm{mmHg}$ was noted between readings, a third measurement was taken with the mean value considered for subsequent analyses. Then, the subjects proceeded to anthropometric measures and familiarization to RE sessions. During the second laboratory visit, resting BP measurements were repeated, and the subjects were further familiarized to the RE sessions. Load determinations were conducted and adjusted up to fourth laboratory visit. Visits 5 and 6 consisted of the TR or CI RE sessions, which were performed in a randomized order. BP and heart rate variability (HRV) responses to MS were evaluated before and after 60 minutes of the RE sessions. Figure 1 illustrates the schematic overview from the experimental sessions.

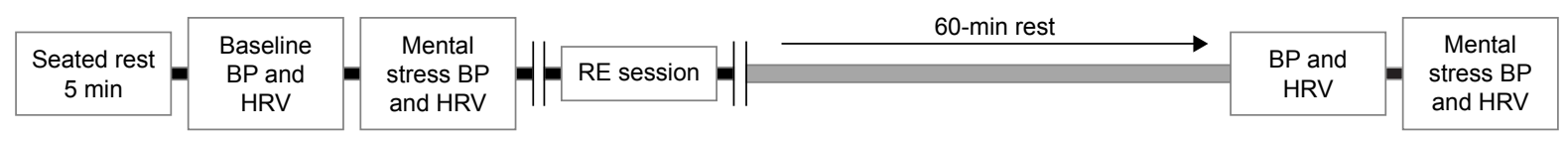

Figure I Schematic diagram of the experimental sessions.

Abbreviations: BP, blood pressure; HRV, heart rate variability; RE, resistance exercise. 


\section{Subjects}

Volunteers were older hypertensive women recruited through flyers, banners, phone calls, and visits to centers of leisure for older adults, hospitals, and churches. In total, 71 subjects were eligible to participate in the present study. The exclusion criteria were as follows: subjects taking hormonal therapy; those with a history of metabolic, neurologic, or cardiovascular diseases other than HTN; those with a history of cardiovascular events; subjects with physical limitations; those with nontreated HTN (ie, systolic BP [SBP] and diastolic BP [DBP] $<140 / 90 \mathrm{mmHg}$, respectively); subjects with obesity (body mass index $\geq 30.0$ ); and subjects taking adrenergic inhibitors or nondihydropyridinic calcium channel blockers. After exclusion ten subjects (age $=71 \pm 5.5$ years; height $=1.5 \pm 0.1 \mathrm{~m}$; weight $=56.1 \pm 9.8 \mathrm{~kg}$ ) participated in this study (six subjects were taking dihydropyridinic calcium channel blockers, six subjects were taking diuretics, and seven subjects were taking angiotensin II receptor blockers). Experimental procedures were in line with the Declaration of Helsinki principles and were approved by the Institutional Review Board at the University of Brasília. All the participants signed an informed consent containing the objectives and procedures as well as possible risks and benefits related to the study. Table 1 presents the subject characteristics, and Figure 2 presents a volunteer recruitment flowchart.

\section{RE load assessment and familiarization}

Exercise loads were assessed over seven REs (ie, seated leg press, seated row, leg extension, seated chest press, leg curl, shoulder abduction, and seated calf raises) by using the perceived effort Omnibus Resistance Exercise Scale (OMNI-RES), according to the procedures described elsewhere. ${ }^{24}$ The rationale of this approach to set the appropriate load used in exercises is that it is an easy, effective, and time-saving procedure supported by a previous research on

Table I Subject characteristics

\begin{tabular}{ll}
\hline Variables & Mean $\pm \mathbf{S D}$ \\
\hline Age (years) & $71.1 \pm 5.5$ \\
Height $(\mathrm{m})$ & $1.5 \pm 0.1$ \\
Weight $(\mathrm{kg})$ & $56.1 \pm 9.8$ \\
BMI $\left(\mathrm{kg} / \mathrm{m}^{2}\right)$ & $24.2 \pm 3.9$ \\
SBP $(\mathrm{mmHg})$ & $119.3 \pm 4.6$ \\
DBP $(\mathrm{mmHg})$ & $68.5 \pm 5.0$ \\
MBP $(\mathrm{mmHg})$ & $85.4 \pm 3.5$ \\
HR $($ bpm) & $69.9 \pm 9.2$ \\
\hline
\end{tabular}

Note: $\mathrm{n}=10$

Abbreviations: BMI, body mass index; DBP, diastolic blood pressure; HR, heart rate; MBP, mean blood pressure; SBP, systolic blood pressure; SD, standard deviation.
RE for older adults. ${ }^{25-27}$ In addition, it also has a practical application for professionals prescribing RE for older adults, as this may be used instead of one repetition maximum or other strength tests. ${ }^{25}$

Except for shoulder abduction that was implemented using dumbbells, all REs were conducted using machines (Rotech Fitness Equipment, Goiania, Brazil). The subjects performed one repetition on each exercise and rated on the scale whether the perceived load was "somewhat easy." If so, the volunteers proceeded to the next exercise; otherwise, the load was adjusted and another repetition was performed after 1 minute of rest, repeating this procedure until the subjects rated the perceived effort as "somewhat easy" for one repetition. It has been demonstrated that one repetition rated "somewhat easy" in OMNI-RES is $\sim 60 \%$ of one maximum repetition. ${ }^{28}$ This load was selected in order to follow RE recommendations for older and hypertensive individuals ${ }^{4,19}$ and thereby induce positive neuromuscular and cardiovascular adaptations. ${ }^{19}$ A print copy of the OMNI-RES was fixed on each exercise machine and was visible to the subjects all the time. As anchoring procedures, the subjects were instructed to create a cognitive visual link using the scale and their memory of muscular efforts. ${ }^{25}$ The

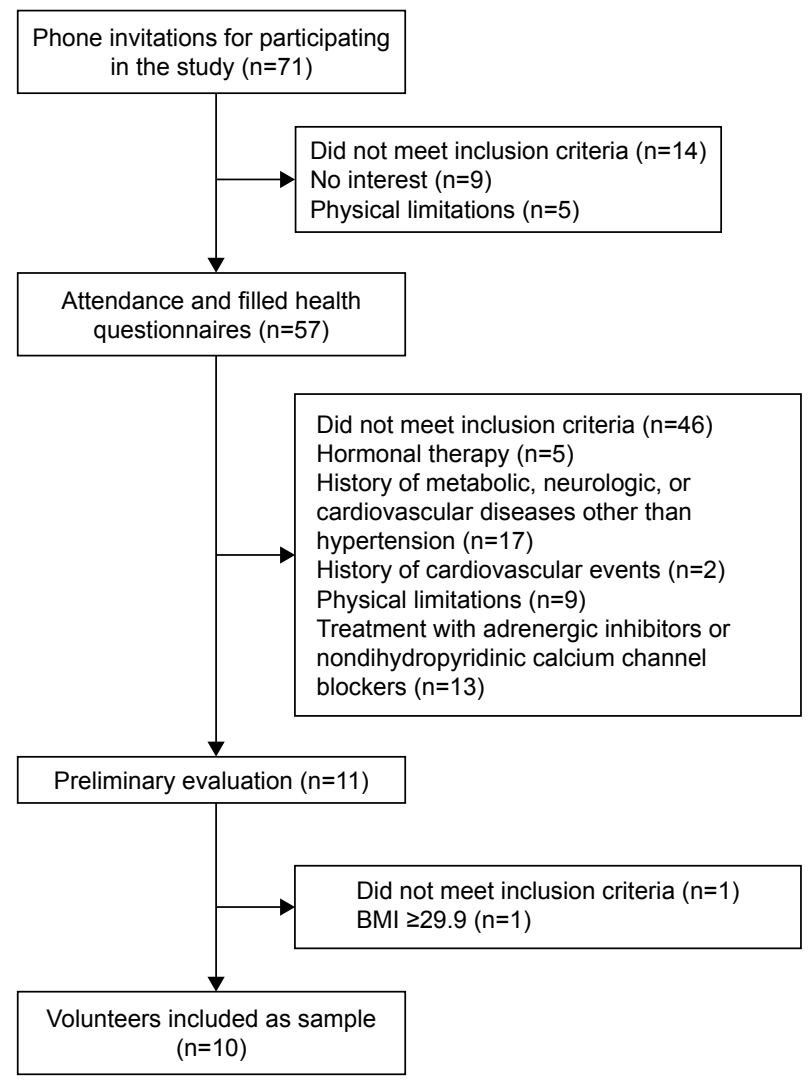

Figure 2 Flowchart of sample recruitment and selection. Abbreviation: BMI, body mass index. 
load values of the RE machines were covered in order to avoid their influence on the perception of subjects.

After exercise load assessment, the volunteers were familiarized to TR or CI RE sessions. They performed one set of 12 repetitions at $50 \%$ of the estimated exercise load at Session 1; two sets of 12 repetitions at $60 \%$ of the estimated exercise load at Session 2; two sets of 12 repetitions at $70 \%$ of the estimated exercise load at Session 3; and three sets of 12 repetitions at $80 \%$ of the estimated exercise load at Session 4. Familiarization TR and CI sessions were separated by 48-72 hours and were performed in a randomized order determined by a statistical software (Decision Analyst STATSтм 2.0, Arlington, VA, USA).

It was previously demonstrated that memory anchoring was equal to exercise anchoring alone or exercise--memory anchoring on reliability ratings of perceived exertion after RE. ${ }^{25}$ Moreover, OMNI-RES applicability was demonstrated in previous studies on older individuals (concurrent and construct validity values in comparison with Borg Scale ranging from 0.79 to 0.91 and 0.94 to 0.97 , respectively), ${ }^{26}$ was recently validated to CI RE arrangement (significant correlation with blood lactate; $r=0.56$ ), and has been shown to be an adequate instrument to manipulate and quantify effort intensity on RE in older women. ${ }^{27,29}$

\section{RE sessions}

In the TR session, the volunteers performed three sets of 12 repetitions for each of the aforementioned exercises, with 1-minute intervals between the sets and exercises. The CI session was performed in 3 laps, consisting of the same exercises, with one set being conducted for each lap, 30-second intervals between exercises and 1 minute between laps. Exercise order and loads were identical for both the arrangements. Immediately after each session, $200 \mathrm{~mL}$ of water was given to the subjects. Thirty minutes after completing the RE sessions, the subjects were presented with an adapted scale of perceived exertion used to quantitate the intensity of RE sessions, following the procedures described elsewhere. ${ }^{30}$ The volunteers were instructed to avoid alcohol and caffeine and not to perform vigorous physical activity during the 24 hours before the experimental sessions. They were also asked to have a light meal 2 hours before each RE session and to maintain their habitual antihypertensive medication routine.

The REs were performed in the following order: seated leg press, seated row, leg extension, chest press, leg curl, shoulder abduction, and seated calf raises. Both the TR and CI sessions were performed in a randomized order at the same time of the morning (between 8 and $11 \mathrm{am}$ ) and were separated by $48-72$ hours.
MS

MS tasks were induced by using a computerized version of the Stroop color-word test and was administrated individually to each participant over a 3-minute period. ${ }^{31}$ Briefly, the study procedure included a slide presentation on a monitor placed in front of the participants. Each slide lasted $\sim 1$ second with the names of colors (ie, "blue," "yellow," and "red") being displayed in different colors from that denoted by the name. The volunteers were instructed to report the color of the letters of the word, as fast as possible, and not the written word. Continuous auditory conflict (a taped voice saying different color names in a headphone) was provided during the test to ensure stress induction, and the recordings were obtained in a temperature-controlled, noise-free room.

The BP was measured by using a mercury-column sphygmomanometer immediately before and at the end of each minute during the test. All of the BP measures were taken by a single trained professional. Within-subjects coefficient of variation for the used device was $6.0 \%$ and $5.7 \%$ for SBP and DBP, respectively. The standard auscultatory method was tested against the automated and previously validated oscillometric method (Microlife BP 3AC1-1). Another sample of 30 independent individuals of both genders rested for 10 minutes, and their BPs were randomly measured by using both the auscultatory and oscillometric methods, the latter conducted by another blinded evaluator. Correlations between the methods were $r=0.90(P<0.001)$ for SBP and $r=0.92$ $(P<0.001)$ for DBP. Furthermore, agreement between these methods was evaluated by using the Bland-Altman method. Mean differences were $3.9 \mathrm{mmHg}(20.2-12.4 \mathrm{mmHg})$ and -3.4 $\mathrm{mmHg}(10.5-17.3 \mathrm{mmHg})$ for SBP and DBP, respectively.

Immediately after the slide presentation, the individuals were asked to report their perceived stress using the following scores: $0=$ not stressful; $1=$ slightly stressful; $2=$ stressful; $3=$ very stressful; and $4=$ extremely stressful. ${ }^{11}$

\section{HRV}

HRV was used to assess autonomic control and was measured by using a cardiac monitor (RS800CX model; Polar Electro Oy, Kempele, Finland), according to the specifications published previously. ${ }^{32}$ Briefly, the subjects wore a heart rate sensor attached to a strap and remained resting in the seated position for 10 minutes with spontaneous breathing prior to pre- and postexercise stress induction. A 5-minute reading was taken before exercise, with the last 3 minutes used for subsequent analyses. HRV was measured during MS both before and after RE sessions. Linear frequencyand time-domain analyses, as well as nonlinear measures of sample entropy (SampEn) were performed by using Kubios 
HRV 2.2 software (Biosignal Analysis and Medical Imaging Group, Joensuu, Finland). Time-domain HRV was expressed as the square root of the mean-squared differences of successive R-R intervals (RMSSD), whereas frequency-domain HRV was assessed by using autoregressive spectral analysis (model order $=16$ ), with HRV decomposed in a low-frequency (LF) band (0.04-0.15 Hz) and a high-frequency (HF) band (0.15-0.4 Hz). The LF/HF ratio of HRV was used as an indicator of sympathovagal balance. Spectral power bands are presented in both absolute $\left(\mathrm{ms}^{2}\right)$ and normalized units $(\mathrm{nu})$.

\section{Statistical analyses}

Reliability of loads determined with OMNI-RES was measured by intraclass correlation coefficient (Cronbach's $\alpha, 95 \%$ confidence interval [CI]). Baseline hemodynamic and autonomic variables prior to RE sessions were compared using repeated measures analysis of variance (ANOVA) with a 2 (session: TR, CI) $\times 2$ (time: pre-RE, post-RE) design for BP response to $\mathrm{MS}$ and a 2 (session: TR, CI) $\times 3$ (time: baseline, during MS pre-RE, during MS post-RE) design for HRV. In addition, the repeated measures ANOVA was performed also to verify $\mathrm{BP}$ responses to MS before exercise (2 [session: TR, $\mathrm{CI}] \times 2$ [BP immediately before MS, BP peak during MS]) and individual perceived stress before and after exercise ( 2 [session: TR, CI] $\times 2$ [time: pre-RE, post-RE]). All the data were presented as mean \pm standard deviation (SD), otherwise noted. Statistical analyses were performed by using Statistical Package for the Social Sciences (version 20.0), with a significance level at $P<0.05$.

\section{Results}

Intraclass correlation coefficients (Cronbach's $\alpha, 95 \% \mathrm{CI}$ ) for loads determined by OMNI-RES demonstrated $r$ values of
0.92 (seated leg press), 0.93 (seated row), 0.98 (leg extension), 0.97 (chest press), 0.87 (leg curl), 0.93 (shoulder abduction), and 0.98 (plantar flexion). No differences in hemodynamic or autonomic variables were observed between RE sessions at baseline or 60 minutes after exercise (Table 2).

Perceived stress induced by Stroop color-word test did not differ within or between experimental sessions at any time (1.7 \pm 0.9 and $1.9 \pm 0.9$ pre- and post-TR sessions, respectively; and $1.8 \pm 0.8$ and $1.7 \pm 0.8$ pre- and post-CI sessions, respectively; session: $P=0.726$; time: $P=0.726$; interaction: $P=0.081)$. Repeated measures ANOVA showed a significant raise in SBP, DBP, and mean $\mathrm{BP}$ (MBP; all $P<0.01)$ during MS before both the RE sessions, showing that the Stroop color-word test as an appropriate stressful tool, with no differences between the sessions.

Figure 3 presents $\mathrm{BP}$ responses immediately before and at each minute of MS (Panels A, C, and E) as well as peak stress-induced BP increases (Panels B, D, and F) both pre- and post-RE sessions. A significant effect of time on SBP (17.4 $\pm 12.8 \Delta$ pre versus $12.5 \pm 9.6 \Delta$ post; $P=0.01)$, DBP (13.7 $\pm 7.1 \Delta$ pre versus $8.8 \pm 4.5 \Delta$ post; $P=0.01)$, and MBP (14.0 $\pm 7.7 \Delta$ pre versus 9.3 $\pm 5.4 \Delta$ post; $P<0.01$ ) was observed. However, no significant interaction was observed, indicating that the RE approaches equally attenuated $\mathrm{BP}$ responses to MS. It was noted that BP readings immediately before the Stroop task did not differ when comparing pre- and post-RE conditions combined, nor between the experimental sessions (ie, TR versus $\mathrm{CI}$ ).

A significant effect of time on LF (nu; 76.8 \pm 15.0 preexercise MS versus 61.4 \pm 19.7 baseline value; $P=0.032$; and $79.1 \pm 9.5$ postexercise MS versus $61.4 \pm 19.7$ baseline value; $P=0.011$ ), $\mathrm{HF}$ (nu; $22.9 \pm 15.1$ pre-exercise MS versus $38.5 \pm 19.7$ baseline value; $P=0.032$; and $20.8 \pm 9.4$ postexercise

Table 2 Pre- and postexercise hemodynamic and autonomic variables in experimental session days (mean \pm SD)

\begin{tabular}{|c|c|c|c|c|c|}
\hline \multirow[t]{2}{*}{ Variables } & \multicolumn{2}{|c|}{ Pre-exercise } & \multicolumn{2}{|c|}{$60 \mathrm{~min}$ postexercise } & \multirow[t]{2}{*}{$P$ (interaction) } \\
\hline & TR RE & CI RE & TR RE & CI RE & \\
\hline $\mathrm{SBP}(\mathrm{mmHg})$ & $120.1 \pm 7.6$ & $117.7 \pm 7.7$ & $120.2 \pm 8.0$ & $116.7 \pm 10.8$ & 0.727 \\
\hline $\mathrm{DBP}(\mathrm{mmHg})$ & $68.9 \pm 5.0$ & $69.6 \pm 5.0$ & $70.7 \pm 4.8$ & $69.6 \pm 6.3$ & 0.158 \\
\hline MBP (mmHg) & $85.9 \pm 4.8$ & $85.6 \pm 4.8$ & $87.2 \pm 5.5$ & $85.3 \pm 7.2$ & 0.220 \\
\hline HR (bpm) & $69.6 \pm 10.1$ & $71.6 \pm 10.7$ & $67.7 \pm 7.8$ & $70.8 \pm 8.4$ & 0.475 \\
\hline $\ln \mathrm{LF}\left(\mathrm{ms}^{2}\right)$ & $4.8 \pm 1.0$ & $4.8 \pm 0.7$ & $5.3 \pm 0.7$ & $5.1 \pm 0.9$ & 0.689 \\
\hline LF (nu) & $60.1 \pm 20.0$ & $61.4 \pm 18.8$ & $61.8 \pm 16.3$ & $70.1 \pm 2 I .1$ & 0.387 \\
\hline In $\mathrm{HF}\left(\mathrm{ms}^{2}\right)$ & $4.3 \pm 0.6$ & $4.3 \pm 0.8$ & $4.7 \pm 0.5$ & $4.2 \pm 0.7$ & 0.095 \\
\hline $\mathrm{HF}(\mathrm{nu})$ & $39.8 \pm 20.0$ & $38.6 \pm 18.8$ & $38.1 \pm 16.3$ & $29.8 \pm 21.1$ & 0.386 \\
\hline LF/HF ratio & $\mathrm{I} . \mathrm{I} \pm 0.2$ & $1.2 \pm 0.3$ & $\mathrm{I} . \mathrm{I} \pm 0.2$ & $1.3 \pm 0.3$ & 0.280 \\
\hline RMSSD & $14.7 \pm 5.2$ & $15.0 \pm 5.5$ & $|7.5 \pm 4|$. & $14.2 \pm 4.6$ & 0.112 \\
\hline SampEn & $I .5 \mathrm{I} \pm 0.22$ & $1.52 \pm 0.28$ & $1.40 \pm 0.13$ & $1.31 \pm 0.19$ & 0.275 \\
\hline
\end{tabular}

Abbreviations: CI RE, circuit-based resistance exercise; DBP, diastolic blood pressure; HF, high frequency; HR, heart rate; LF, low frequency; In, logarithmic normalization; MBP, mean blood pressure; nu, normalized units; RMSSD, square root of the mean-squared differences of successive R-R intervals; SampEn, sample entropy; SBP, systolic blood pressure; SD, standard deviation; TR RE, traditional resistance exercise. 
A
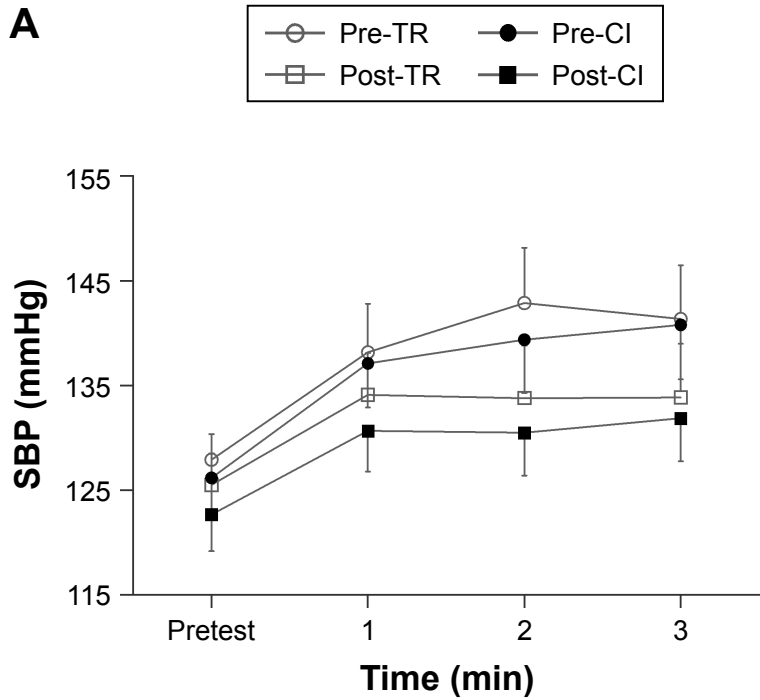

C
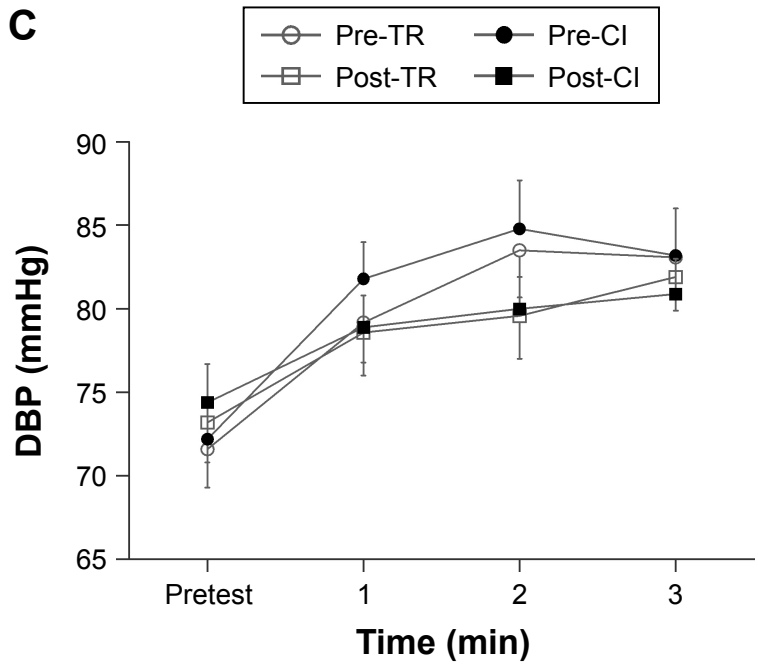

$\mathbf{E}$
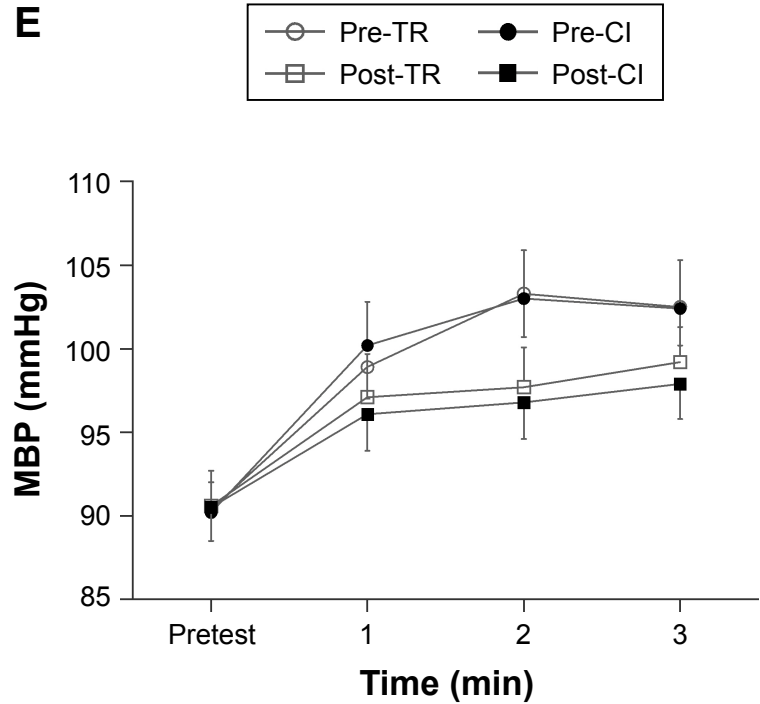

B

Pre-exercise $\square$ Postexercise

Session: $P=0.843$

Time: $P=0.014$

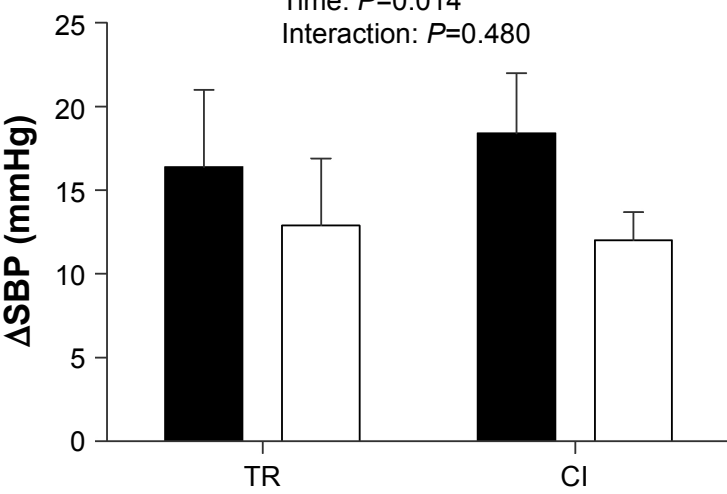

RE session

D

Pre-exercise $\square$ Postexercise

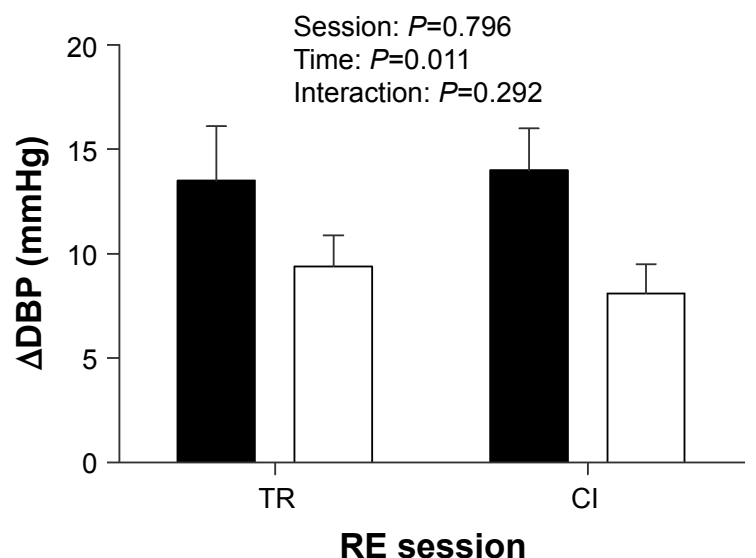

$\mathbf{F}$

Pre-exercise $\square$ Postexercise

Session: $P=0.656$

Time: $P=0.007$

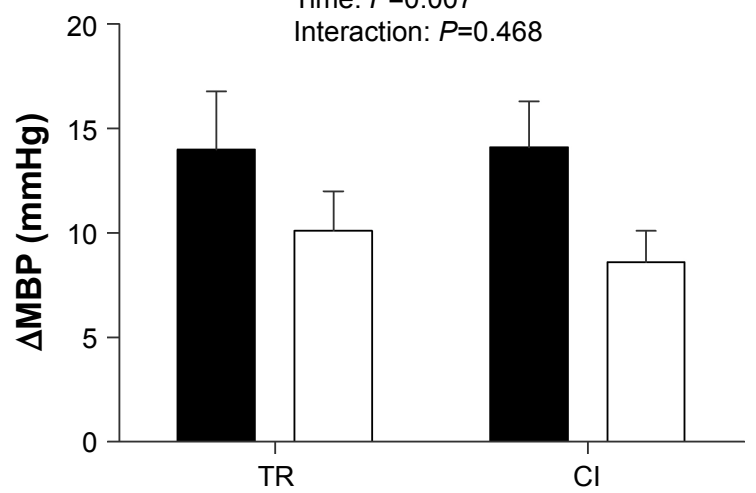

RE session

Figure 3 Time course for SBP, DBP, and MBP responses and their changes (SBP, Panels $\mathbf{A}$ and $\mathbf{B}$; DBP, Panels $\mathbf{C}$ and $\mathbf{D}$; and MBP, Panels $\mathbf{E}$ and $\mathbf{F}$ ) during MS before and 60 min after TR and CI RE sessions.

Note: Values are mean \pm standard error of mean.

Abbreviations: Cl, circuit-based; DBP, diastolic blood pressure; MBP, mean blood pressure; MS, mental stress; RE, resistance exercise; SBP, systolic blood pressure; SD, standard deviation; TR, traditional. 
Table 3 Mean \pm SD of HRV components at baseline and during MS by Stroop task performed pre- and post-TR RE session and preand post-CI RE session

\begin{tabular}{|c|c|c|c|c|c|c|c|}
\hline & In LF $\left(\mathrm{ms}^{2}\right)$ & LF (nu) & In HF $\left(\mathrm{ms}^{2}\right)$ & HF (nu) & LF/HF & RMSSD & SampEn \\
\hline \multicolumn{8}{|l|}{ TR } \\
\hline Baseline & $5.0 \pm 1.1$ & $62.2 \pm 21.4$ & $4.4 \pm 0.6$ & $37.7 \pm 21.3$ & $1.1 \pm 0.2$ & $|5| \pm 5.5$. & $1.62 \pm 0.23$ \\
\hline MS pre-exercise & $5.4 \pm 0.8$ & $79.8 \pm 8.6$ & $3.9 \pm 0.5$ & $20.2 \pm 8.5$ & $1.4 \pm 0.1$ & $13.6 \pm 3.0$ & $\mid .31 \pm 0.21$ \\
\hline MS postexercise & $5.4 \pm 0.9$ & $78.5 \pm 9.1$ & $4.0 \pm 0.5$ & $21.4 \pm 9.0$ & $1.3 \pm 0.1$ & $14.6 \pm 3.5$ & $1.28 \pm 0.18$ \\
\hline \multicolumn{8}{|l|}{$\mathrm{Cl}$} \\
\hline Baseline & $4.7 \pm 0.9$ & $60.6 \pm 19.4$ & $4.2 \pm 0.8$ & $39.4 \pm 19.4$ & $1.1 \pm 0.2$ & $14.5 \pm 5.8$ & $1.54 \pm 0.19$ \\
\hline MS pre-exercise & $5.2 \pm 0.8$ & $73.9 \pm 19.7$ & $4.0 \pm 0.6$ & $25.7 \pm 20.0$ & $1.3 \pm 0.3$ & $13.4 \pm 4.5$ & $1.26 \pm 0.19$ \\
\hline MS postexercise & $5.4 \pm 0.9$ & $79.8 \pm 10.5$ & $3.9 \pm 0.7$ & $20.2 \pm 10.4$ & $1.4 \pm 0.2$ & $14.3 \pm 3.8$ & $1.09 \pm 0.26$ \\
\hline \multicolumn{8}{|l|}{$P$ (ANOVA) } \\
\hline Session & 0.451 & 0.409 & 0.488 & 0.446 & 0.892 & 0.739 & 0.134 \\
\hline Time & $0.029 *$ & $0.006 *$ & 0.230 & $0.006 *$ & $0.004 *$ & 0.611 & $<0.001 *$ \\
\hline Interaction & 0.754 & 0.651 & 0.456 & 0.680 & 0.581 & 0.951 & 0.518 \\
\hline
\end{tabular}

Note: *Significant effect of time $(P<0.05)$.

Abbreviations: ANOVA, analysis of variance; Cl, circuit-based; HF, high frequency; HRV, heart rate variability; LF, low frequency; In, logarithmic normalization; MS, mental stress; nu, normalized units; RE, resistance exercise; RMSSD, square root of the mean-squared differences of successive R-R intervals; SampEn, sample entropy; SD, standard deviation; TR, traditional.

MS versus $38.5 \pm 19.7$ baseline value; $P=0.011$ ), $\mathrm{LF} / \mathrm{HF}$ ratio (1.4 \pm 0.2 pre-exercise MS versus $1.1 \pm 0.3$ baseline value; $P=0.025$; and $1.4 \pm 0.2$ postexercise MS versus $1.1 \pm 0.3$ baseline value; $P=0.010)$, and SampEn ( $1.28 \pm 0.20$ pre-exercise MS versus $1.58 \pm 0.21$ baseline value; $P=0.015$; and $1.19 \pm 0.23$ postexercise MS versus $1.58 \pm 0.21$ baseline value; $P=0.004)$ was observed, showing significant changes from baseline to MS pre-RE and also from baseline to MS post-RE (Table 3). Interestingly, a significant effect of time on log-normalized low-frequency (ln LF; $\mathrm{ms}^{2} ; 5.3 \pm 0.8$ pre-exercise MS versus $4.8 \pm 1.0$ baseline value; $P=0.023$ ) was also observed, showing a significant change only from baseline to MS pre-RE, but not from baseline to MS post-RE sessions. The comparison of $\ln \operatorname{LF}\left(\mathrm{ms}^{2}\right)$ postexercise with baseline values did not show significant differences. A time effect was absent for ln HF $\left(\mathrm{ms}^{2}\right)$ and RMSSD. Session and interaction effects were not observed for any of the HRV variables.

\section{Discussion}

The acute effects of aerobic exercise on BP reactivity to MS have been extensively studied. ${ }^{13}$ However, although RE has been recommended for older hypertensive individuals, it is unknown whether this mode of exercise can provide similar benefits on the response to MS. The responses of different $\mathrm{RE}$ methods to BP reactivity would also be of interest, as TR and CI methods have been commonly used in RE studies for hypertensive individuals. ${ }^{21,22}$ The main findings of the present study provide the novel insight that both TR and $\mathrm{CI}$ RE sessions attenuate $\mathrm{BP}$ reactivity to MS in older hypertensive women, with no different effects between the sessions. These observations were followed by a reduction in sympathetic neural activity as evaluated by using HRV during MS, which might in part explain the attenuated BP response. These results have potential relevant clinical significance and extend previous evidence that recommends $\mathrm{RE}$ as an important component of physical activity programs designed for older hypertensive subjects. ${ }^{19}$

The results from the present study corroborate previous studies that demonstrated that a single session of aerobic exercise attenuates BP responses to MS. ${ }^{11,12}$ It was observed that reductions in $\mathrm{BP}$ reactivity were similar to those reported in a meta-analysis by Hamer et al. ${ }^{13}$ These authors reported mean reductions of 3.0 and $3.9 \mathrm{mmHg}$ for SBP and DBP, respectively, following aerobic exercise sessions. The present results similarly indicated reductions of 3.5 (SBP) and $4.0 \mathrm{mmHg}$ (DBP) following TR RE sessions and 6.4 (SBP) and $5.9 \mathrm{mmHg}$ (DBP) after CI RE sessions, but with no significant differences between them. To the authors' knowledge, only one study by Moreira et $\mathrm{al}^{23}$ that included $\mathrm{RE}$ as part of a concurrent session designed in a CI manner reported an attenuated BP response to a cold pressor test following exercise; however, the BP rise in vascular tests in response to cold pressor testing occurred by different mechanisms from that elicited by Stroop color-word test, and these results may not occur if other stressors were employed. The present observations expand previous findings and suggest that RE per se could diminish the BP response to MS in older hypertensive women.

No differences in postexercise BP responses were observed between TR and CI even though these two approaches had equivalent loads and volume. In addition, the ratings of perceived exertion did not differ between the 
two approaches to RE $(3.7 \pm 1.1$ for TR and 3.4 \pm 1.2 for CI; $P=0.193)$. CI RE has become a popular method for older adults and has been suggested to be advantageous, given its dynamic and time-saving nature. In fact, the CI session was accomplished in approximately two-thirds of the time required for the TR session $(29.9 \pm 0.8$ minutes for TR and $20.2 \pm 0.8$ minutes for $\mathrm{CI})$. Therefore, it can be argued that the CI approach is an equally effective and more efficient RE method for older hypertensive women as it promotes similar attenuation in BP reactivity when compared with TR, but it is completed in a shorter duration. In addition to the potential advantages when considering adherence, faster completion of CI might help to facilitate other modes such as aerobic and flexibility exercises in a typical exercise session.

In the present study, attenuation in BP reactivity might partially be explained by an acute RE-induced lessening in sympathetic activity during MS. This was supported by an absence of response from LF spectral band $\left(\mathrm{ms}^{2}\right)$ during MS post-RE relative to baseline, while a significant rise in this frequency band was evident during MS before exercise. The results from the present study were in agreement with the observations of Brownley et al, ${ }^{12}$ who showed that diminished sympathetic reactivity (reductions in norepinephrine and a rise in pre-ejection period) was responsible for up to $64 \%$ of the attenuation found in MBP during MS after a bout of aerobic exercise. However, previous reports regarding catecholamine levels and BP reactivity after dynamic exercise are controversial. Péronnet et $\mathrm{al}^{33}$ reported a reduction in epinephrine levels after exercise during an MS test that was not accompanied by reductions in BP reactivity; Ebbesen et $\mathrm{al}^{18}$ found the opposite results, showing a reduction in BP reactivity to MS and a maintenance of circulating catecholamines.

It is possible that the attenuated BP response observed during MS post-RE could also be mediated, in part, by a vasodilator response. This is supported by the findings of West et al, ${ }^{16}$ who demonstrated a reduction in peripheral vascular resistance following a bout of exercise together with a reduction in $\mathrm{BP}$ reactivity to stress. In addition, the results of Brownley et $\mathrm{al}^{12}$ corroborate this hypothesis. They reported a significant rise in $\beta$ - 2 adrenergic responsiveness after performing an exercise bout, which is demonstrated by a reduction in isoproterenol vasodilator dose necessary to diminish peripheral vascular resistance by $50 \%$, suggesting that an adrenergic-mediated $(\beta-2)$ vasodilation is one of the mechanisms responsible for BP attenuation during stress after exercise. Nonetheless, this concept is challenged by previous studies reporting that reductions in stroke volume and cardiac output but not peripheral vascular resistance are responsible for a diminished $\mathrm{BP}$ response to $\mathrm{MS}$ following exercise. ${ }^{11,34}$ The present results extend the previous studies supporting cardiovascular benefits of RE. ${ }^{4,19}$ It was observed that RE may reduce the sympathetic response to stress, limiting individual exposure to high levels of sympathetic activity. It was noted that high levels of sympathetic activity reflected by HRV are associated with a variety of adverse cardiovascular conditions including myocardial infarction, transient myocardial ischemia, congestive heart failure, and mortality. ${ }^{35}$ In addition, as stated by Hamer et al $^{13}$ repeated, cumulative bouts of exercise are associated with cardiovascular health benefits, in part through its effect on lowering $\mathrm{BP}$ response to MS. This is important given that individuals who exhibit high BP responses to MS may be at a risk of developing diseases such as HTN, ${ }^{6}$ silent cerebrovascular disease, ${ }^{36}$ stroke, ${ }^{37}$ cardiovascular mortality, ${ }^{38}$ and transient endothelial dysfunction. ${ }^{39}$

Volunteers enrolled in this investigation were older women who had HTN, two conditions associated with a high cardiovascular risk. Older adults, for example, showed a high SBP reactivity to stress when compared to young. ${ }^{40}$ As demonstrated by Everson et al, ${ }^{37}$ each $1 \mathrm{mmHg}$ rise in SBP during a stressful situation corresponded to an $1 \%$ rise in stroke risk in an 11-year prospective study. Similarly, Carroll et $\mathrm{al}^{38}$ observed that every $1-\mathrm{SD}$ rise in SBP reactivity during stress was associated with a 3\% elevation in cardiovascular mortality among 431 individuals followed up for 16 years. The present results may also have clinical significance for hypertensive individuals, as their conditions already represent an elevated cardiovascular risk that could be minimized by regular REs. Thus, the results of the present study supported guidelines from professional organizations regarding $\mathrm{RE}$ for older adults ${ }^{41}$ and for the management of HTN. ${ }^{4}$

Pre-exercise BP levels were not diminished after 60 minutes of exercise in the present study, indicating the absence of postexercise hypotension. Despite evidence regarding the occurrence of post-RE hypotension in older ${ }^{42,43}$ and hypertensive individuals, ${ }^{44,45}$ it is important to consider that the magnitude of BP changes in the postexercise period may directly be associated with higher pre-exercise BP levels, ${ }^{46}$ which was not the case in the present sample. Moreover, as suggested by West et $\mathrm{al}^{16}$ and observed in previous reports, ${ }^{47,48}$ anticipation of the upcoming stress test may prevent any significant BP reductions after exercise. The present results showed that even in the absence of postexercise hypotension, performing a single bout of RE may reduce cardiovascular risk by minimizing BP and sympathetic responses to stressful situations.

A lack of information exists concerning the effects of RE on $\mathrm{BP}$ response to a MS. The present study reported for the first 
time that RE can be used as a coping strategy to attenuate BP response to an MS test. Significant reductions in SBP, DBP, and MBP were shown after performing TR and CI sessions of RE, with no difference between the methods. The sessions were prescribed with volume and intensity commonly used in RE programs designed for older subjects (three sets with an intensity corresponding to a "somewhat easy" load on the OMNI-RES, a time-saving, efficient, and safe strategy on load determination). These findings were relevant considering the health risks associated with a hyperreactive BP response, ${ }^{36,37}$ showing that RE should be performed even in the absence of postexercise hypotension. This is important for hypertensive individuals, who present an elevated cardiovascular risk compared with their normotensive counterparts. ${ }^{1}$

\section{Limitations}

The present study has several limitations. For example, HRV is an indirect measure of autonomic nervous activity that may not be sensitive enough to reflect changes in autonomic modulation during MS. ${ }^{49,50}$ However, a series of previous studies employed HRV as a measure of cardiac autonomic control during MS, ${ }^{51-53}$ and the results from the present study suggested a well-characterized response of sympathetic activation during stress. Furthermore, the volunteers were women with well-controlled HTN, and thus, the present observations cannot necessarily be extrapolated to those with uncontrolled HTN and to men. Future studies should be conducted in this regard.

\section{Conclusion}

The findings from the present study suggested that both TR and CI RE sessions acutely attenuate BP responses to MS in older hypertensive women, with no differences between the two approaches. These responses may partially be explained by the reduced sympathetic response during MS after exercise. These results have clinical implications in that they reinforce previous evidence that recommends RE as an important component of physical activity programs designed for older hypertensive subjects.

\section{Acknowledgments}

The authors thank the study participants. This work was supported by National Council for Scientific and Technological Development (grants 487622/2012-0 and 307630/2013-7) and by University of Brasília. The authors thank the Federal District Research Support Foundation (FAPDF) for funding the publication fee. Preliminary results of this work were published as an abstract in "ISAN 2015: First International Joint Meeting ISAN - AAS - EFAS - JSNR
2015," held in Stresa, Italy. The abstract is referenced as Autonomic Neuroscience: basic and clinic, 2015. v. 192. p. 111. The text is available online and can be found in http://www.autonomicneuroscience.com/article/S15660702(15)00246-5/abstract.

\section{Disclosure}

The authors report no conflicts of interest in this work.

\section{References}

1. Aronow WS, Fleg JL, Pepine CJ, et al. ACCF/AHA 2011 expert consensus document on hypertension in the elderly: a report of the American College of Cardiology Foundation Task Force on clinical expert consensus documents developed in collaboration with the American Academy of Neurology, American Geriatrics Society, American Society for Preventive Cardiology, American Society of Hypertension, American Society of Nephrology, Association of Black Cardiologists, and European Society of Hypertension. J Am Coll Cardiol. 2011;57(20):2037-2114.

2. Chobanian AV, Bakris GL, Black HR, et al. The Seventh Report of the Joint National Committee on Prevention, Detection, Evaluation, and Treatment of High Blood Pressure: the JNC 7 report. JAMA. 2003; 289(19):2560-2571.

3. Lima R, Wofford M, Reckelhoff JF. Hypertension in postmenopausal women. Curr Hypertens Rep. 2012;14(3):254-260.

4. Pescatello LS, Franklin BA, Fagard R, et al. American College of Sports Medicine position stand. Exercise and hypertension. Med Sci Sports Exerc. 2004;36(3):533-553.

5. Pickering TG. Mental stress as a causal factor in the development of hypertension and cardiovascular disease. Curr Hypertens Rep. 2001; 3(3):249-254.

6. Chida Y, Steptoe A. Greater cardiovascular responses to laboratory mental stress are associated with poor subsequent cardiovascular risk status a meta-analysis of prospective evidence. Hypertension. 2010; 55(4):1026-1032.

7. Matthews KA, Owens JF, Kuller LH, Sutton-Tyrrell K, Lassila HC, Wolfson SK. Stress-induced pulse pressure change predicts women's carotid atherosclerosis. Stroke. 1998;29(8):1525-1530.

8. Lindvall K, Kahan T, de Faire U, Ostergren J, Hjemdahl P. Stressinduced changes in blood pressure and left ventricular function in mild hypertension. Clin Cardiol. 1991;14(2):125-132.

9. Tsai PS, Yucha CB, Nichols WW, Yarandi H. Hemodynamics and arterial properties in response to mental stress in individuals with mild hypertension. Psychosom Med. 2003;65(4):613-619.

10. Cardillo C, Kilcoyne CM, Cannon RO, Panza JA. Impairment of the nitric oxide-mediated vasodilator response to mental stress in hypertensive but not in hypercholesterolemic patients. J Am Coll Cardiol. 1998;32(5):1207-1213.

11. Vianna LC, Silva BM, Nóbrega ACL. Sex differences in blood pressure responses to mental stress are abolished after a single bout of exercise: underlying hemodynamic mechanisms. J Physiol Sci. 2014;64(3):213-219.

12. Brownley KA, Hinderliter AL, West SG, Girdler SS, Sherwood A, Light KC. Sympathoadrenergic mechanisms in reduced hemodynamic stress responses after exercise. Med Sci Sports Exerc. 2003;35(6):978-986.

13. Hamer M, Taylor A, Steptoe A. The effect of acute aerobic exercise on stress related blood pressure responses: a systematic review and meta-analysis. Biol Psychol. 2006;71(2):183-190.

14. Sales AR, Fernandes IA, Rocha NG, et al. Aerobic exercise acutely prevents the endothelial dysfunction induced by mental stress among subjects with metabolic syndrome: the role of shear rate. Am J Physiol Heart Circ Physiol. 2014;306(7):H963-H971.

15. Boone JB Jr, Probst MM, Rogers MW, Berger R. Postexercise hypotension reduces cardiovascular responses to stress. J Hypertens. 1993;11(4):449-453. 
16. West SG, Brownley KA, Light KC. Postexercise vasodilatation reduces diastolic blood pressure responses to stress. Ann Behav Med. 1998;20(2):77-83.

17. Hamer M, Jones J, Boutcher SH. Acute exercise reduces vascular reactivity to mental challenge in offspring of hypertensive families. J Hypertens. 2006;24(2):315-320.

18. Ebbesen BL, Prkachin KM, Mills DE, Green HJ. Effects of acute exercise on cardiovascular reactivity. J Behav Med. 1992;15(5):489-507.

19. Williams MA, Haskell WL, Ades PA, et al. Resistance exercise in individuals with and without cardiovascular disease: 2007 update a scientific statement from the american heart association council on clinical cardiology and council on nutrition, physical activity, and metabolism. Circulation. 2007;116(5):572-584.

20. Teixeira L, Ritti-Dias RM, Tinucci T, Júnior DM, Forjaz CLM. Postconcurrent exercise hemodynamics and cardiac autonomic modulation. Eur J Appl Physiol. 2011;111(9):2069-2078.

21. Harris KA, Holly RG. Physiological response to circuit weight training in borderline hypertensive subjects. Med Sci Sports Exerc. 1987; 19(3):246-252.

22. Moraes MR, Bacurau RF, Simoes HG, et al. Effect of 12 weeks of resistance exercise on post-exercise hypotension in stage 1 hypertensive individuals. J Hum Hypertens. 2012;26(9):533-539.

23. Moreira SR, Lima RM, Silva KE, Simoes HG. Combined exercise circuit session acutely attenuates stress-induced blood pressure reactivity in healthy adults. Braz J Phys Ther. 2014;18(1):38-46.

24. Robertson RJ, Goss FL, Rutkowski J, et al. Concurrent validation of the OMNI perceived exertion scale for resistance exercise. Med Sci Sports Exerc. 2003;35(2):333-341.

25. Lagally KM, Costigan EM. Anchoring procedures in reliability of ratings of perceived exertion during resistance exercise. Percept Mot Skills. 2004;98(3 Pt 2):1285-1295.

26. Gearhart RF Jr, Lagally KM, Riechman SE, Andrews RD, Robertson RJ. Strength tracking using the OMNI resistance exercise scale in older men and women. $J$ Strength Cond Res. 2009;23(3):1011-1015.

27. Ferreira SS, Krinski K, Alves RC, et al. The use of session RPE to monitor the intensity of weight training in older women: acute responses to eccentric, concentric, and dynamic exercises. J Aging Res. 2014;2014:749317.

28. Lagally KM, Robertson RJ. Construct validity of the OMNI resistance exercise scale. J Strength Cond Res. 2006;20(2):252-256.

29. Aniceto RR, Ritti-Dias RM, Dos Prazeres TM, Farah BQ, de Lima FF, do Prado WL. Rating of perceived exertion during circuit weight training: a concurrent validation study. J Strength Cond Res. 2015; 29(12):3336-3342.

30. Sweet TW, Foster C, McGuigan MR, Brice G. Quantitation of resistance training using the session rating of perceived exertion method. J Strength Cond Res. 2004;18(4):796-802.

31. Stroop JR. Studies of interference in serial verbal reactions. $J$ Exp Psychol. 1935;18(6):643-662.

32. Task Force of the European Society of Cardiology and the North American Society of Pacing and Electrophysiology. Heart rate variability. Standards of measurement, physiological interpretation, and clinical use. Task Force of the European Society of Cardiology and the North American Society of Pacing and Electrophysiology. Eur Heart J. 1996;17(3):354-381.

33. Péronnet F, Massicotte D, Paquet JE, Brisson G, de Champlain J. Blood pressure and plasma catecholamine responses to various challenges during exercise-recovery in man. Eur J Appl Physiol Occup Physiol. 1989;58(5):551-555.

34. Neves FJ, Carvalho AC, Rocha NG, et al. Hemodynamic mechanisms of the attenuated blood pressure response to mental stress after a single bout of maximal dynamic exercise in healthy subjects. Braz J Med Biol Res. 2012;45(7):610-616.
35. Montano N, Porta A, Cogliati C, et al. Heart rate variability explored in the frequency domain: a tool to investigate the link between heart and behavior. Neurosci Biobehav Rev. 2009;33(2):71-80.

36. Waldstein SR, Siegel EL, Lefkowitz D, et al. Stress-induced blood pressure reactivity and silent cerebrovascular disease. Stroke. 2004; 35(6):1294-1298.

37. Everson SA, Lynch JW, Kaplan GA, Lakka TA, Sivenius J, Salonen JT. Stress-induced blood pressure reactivity and incident stroke in middleaged men. Stroke. 2001;32(6):1263-1270.

38. Carroll D, Ginty AT, Der G, Hunt K, Benzeval M, Phillips AC. Increased blood pressure reactions to acute mental stress are associated with 16-year cardiovascular disease mortality. Psychophysiology. 2012; 49(10): 1444-1448

39. Ghiadoni L, Donald AE, Cropley M, et al. Mental stress induces transient endothelial dysfunction in humans. Circulation. 2000;102(20): 2473-2478.

40. Uchino BN, Birmingham W, Berg CA. Are older adults less or more physiologically reactive? A meta-analysis of age-related differences in cardiovascular reactivity to laboratory tasks. J Gerontol B Psychol Sci Soc Sci. 2010;65(2):154-162.

41. American College of Sports Medicine, Chodzko-Zajko WJ, Proctor DN, et al. American College of Sports Medicine position stand. Exercise and physical activity for older adults. Med Sci Sports Exerc. 2009;41(7): 1510-1530.

42. Mota MR, de Oliveira RJ, Dutra MT, et al. Acute and chronic effects of resistive exercise on blood pressure in hypertensive elderly women. J Strength Cond Res. 2013;27(12):3475-3480.

43. Queiroz AC, Kanegusuku H, Chehuen MR, et al. Cardiac work remains high after strength exercise in elderly. Int J Sports Med. 2013; 34(5):391-397.

44. Brito Ade F, de Oliveira CV, Brasileiro-Santos Mdo S, Santos Ada C. Resistance exercise with different volumes: blood pressure response and forearm blood flow in the hypertensive elderly. Clin Interv Aging. 2014;9:2151-2158.

45. Scher LM, Ferriolli E, Moriguti JC, Scher R, Lima NK. The effect of different volumes of acute resistance exercise on elderly individuals with treated hypertension. J Strength Cond Res. 2011;25(4):1016-1023.

46. Fisher MM. The effect of resistance exercise on recovery blood pressure in normotensive and borderline hypertensive women. J Strength Cond Res. 2001;15(2):210-216.

47. Rejeski WJ, Gregg E, Thompson A, Berry M. The effects of varying doses of acute aerobic exercise on psychophysiological stress responses in highly trained cyclists. J Sport Exerc Psychol. 1991;13:188-199.

48. Rejeski WJ, Thompson A, Brubaker PH, Miller HS. Acute exercise: buffering psychosocial stress responses in women. Health Psychol. 1992;11(6):355-362.

49. Sloan R, Korten J, Myers MM. Components of heart rate reactivity during mental arithmetic with and without speaking. Physiol Behav. 1991;50(5):1039-1045.

50. Sloan RP, Shapiro P, Bagiella E, Bigger J, Lo E, Gorman J. Relationships between circulating catecholamines and low frequency heart period variability as indices of cardiac sympathetic activity during mental stress. Psychosom Med. 1996;58(1):25-31.

51. Uchino BN, Uno D, Holt-Lunstad J, Flinders JB. Age-related differences in cardiovascular reactivity during acute psychological stress in men and women. J Gerontol B Psychol Sci Soc Sci. 1999;54(6):P339-P346.

52. Langewitz W, Rüddel H, Schächinger H, et al. Changes in sympathetic and parasympathetic cardiac activation during mental load: an assessment by spectral analysis of heart rate variability. Homeost Health Dis. 1990;33(1-2):23-33.

53. Bernardi L, Wdowczyk-Szulc J, Valenti C, et al. Effects of controlled breathing, mental activity and mental stress with or without verbalization on heart rate variability. J Am Coll Cardiol. 2000;35(6):1462-1469. 
Clinical Interventions in Aging

\section{Publish your work in this journal}

Clinical Interventions in Aging is an international, peer-reviewed journal focusing on evidence-based reports on the value or lack thereof of treatments intended to prevent or delay the onset of maladaptive correlates of aging in human beings. This journal is indexed on PubMed Central, MedLine,

CAS, Scopus and the Elsevier Bibliographic databases. The manuscript management system is completely online and includes a very quick and fair peer-review system, which is all easy to use. Visit http://www.dovepress. $\mathrm{com} /$ testimonials.php to read real quotes from published authors.

Submit your manuscript here: http://www.dovepress.com/clinical-interventions-in-aging-journal 